\title{
Narrativa
}

\section{Tutto già globalizzato? La letteratura italiana in Polonia dopo il 1989}

\section{Hanna Serkowska e Katarzyna Misiewicz}

\section{(2) OpenEdition}

\section{Journals}

\section{Edizione digitale}

URL: https://journals.openedition.org/narrativa/828

DOI: $10.4000 /$ narrativa. 828

ISSN: 2804-1224

\section{Editore}

Presses universitaires de Paris Nanterre

\section{Edizione cartacea}

Data di pubblicazione: 1 décembre 2016

Paginazione: 89-99

ISBN: 978-2-84016-266-7

ISSN: $1166-3243$

\section{Notizia bibliografica digitale}

Hanna Serkowska e Katarzyna Misiewicz, «Tutto già globalizzato? La letteratura italiana in Polonia dopo il 1989», Narrativa [Online], 38 | 2016, online dal 01 janvier 2022, consultato il 22 février 2022. URL: http://journals.openedition.org/narrativa/828 ; DOI: https://doi.org/10.4000/narrativa.828

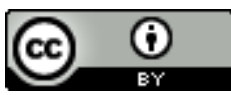

Narrativa est mise à disposition selon les termes de la Licence Creative Commons Attribution 4.0 International. 


\section{Tutto già globalizzato? \\ La letteratura italiana in Polonia dopo il 1989}

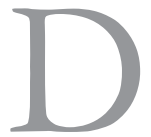

iversamente da quanto succede in molti paesi europei, in Polonia il numero degli iscritti a corsi di italiano non ha registrato flessioni, da quando, oltre quarant'anni fa, sono state create le prime facoltà di italianistica. Si nota perfino un costante aumento dei dipartimenti di italianistica. Tuttavia, specie negli atenei privati, il livello di insegnamento e le competenze degli studenti non sono altissimi, i discenti usano spesso e volentieri testi in traduzione polacca. Inoltre, i programmi di insegnamento restano immutati da decenni; la letteratura proposta non oltrepassa la soglia degli anni '80 e la scelta di autori contemporanei è determinata dai fattori specificati nel saggio di Giuliana Benvenuti incluso in questo volume: gli interessi del docente, lo stile semplice di un testo e sua la capacità di trasmettere l'immagine dell'Italia. Di conseguenza nell'insegnamento dell'italiano fuori Italia si va formando un canone didattico globalizzato, diverso dal canone delle traduzioni dall'italiano, il quale sembra variare da paese a paese.

Quanto ai testi italiani in traduzione polacca, a tutt'oggi non esiste in Polonia una bibliografia unica. Esistono bibliografie frammentarie, stese secondo l'argomento ${ }^{1}$, l'autore ${ }^{2}$, o la traduzione ${ }^{3}$. Già nel 1988, nel primo numero del Bollettino di Studi Italiani, Irena Bednarz affermava la necessità di realizzare una

1. ŻAboklicki, Krzysztof, L'italianistica in Polonia, in Graciotti, Sante, ŻabokLICKI, Krzysztof, La polonistica in Italia e l'italianistica in Polonia 1945-1979, Wrocław, Ossolineum, 1983.

2. Preisner, Walerian, Dante i jego dzieła w Polsce: Bibliografia krytyczna z historycznym wstepem, Warszawa, PWN, 1957.

3. Bednarz, Irena, "Bibliografia przekładów z literatury włoskiej od 1995r", in SAlWa, Piotr (a cura di), Historia literatury wtoskiej, II voll., WarszaWa, Semper, 1997, vol. II, pp. 387-405; BEDNARZ, Irena, "Bibliografia literatury włoskiej XX wieku", in Ugniewska, Joanna, Historia literatury wtoskiej XXw., Warszawa, PWN, 2001 (ediz. aggiornata), pp. 355-446. 
Polska bibliografia italianistyczna (Bibliografia degli italica polacchi) e procedeva alla schedatura gli anni 1985-1987: in tutto 339 voci ripartite tra linguistica, letteratura, teatro, film, storia e storia dell'arte. Se nelle sillogi di letteratura europea si rinvengono solo bibliografie in forma di appendice ${ }^{4}$, il Dipartimento di italianistica dell'Università di Varsavia, dal $1993^{5}$, invia regolarmente gli "italica" polacchi (comprese le informazioni sulle traduzioni dall'italiano pubblicate in Polonia) all'annuario di bibliografia italiana di portata mondiale, Bibliografia Generale della Lingua e della Letteratura Italiana ${ }^{6}$. La Polska Bibliografia Literacka online (PBL, Bibliografia letteraria polacca) è parziale e copre gli anni 1988-20027 . Tra le varie sezioni, sotto "letteratura italiana" vi sono attualmente circa quattromila voci, che mescolano però testi tradotti, recensioni, segnalazioni, interviste, saggi e articoli. A una prima scorsa delle pagine dedicate alla letteratura italiana in Polonia si rivelano pesanti lacune e omissioni, al punto che risulta insondabile il criterio di selezione seguito dal sito, e le informazioni reperibili rischiano di depistare il ricercatore piuttosto che ragguagliarlo. Deludente per i nostri fini, e solo parzialmente utile, appare il NUKAT, Narodowy Uniwersalny Katalog Centralny (Catalogo Centrale Nazionale ${ }^{8}$ ) che copre gli anni più recenti, e inizia là dove si ferma la PBL. Esso dovrebbe svolgere le stesse funzioni del catalogo del servizio bibliotecario italiano opac.sbn, offrendo a chiunque l'informazione sulla disponibilità del libro in biblioteche polacche. Nato soltanto nel 2002 per integrare le informazioni sul contenuto delle biblioteche, è uno strumento in via di costruzione, anch'esso parziale. Consultando invece i cataloghi della Biblioteca Nazionale ${ }^{9}$ o della biblioteca IBL PAN ${ }^{10}$, inserito il nome dell'autore o del traduttore, appaiono tutti i riferimenti aggiornati. Anche la biblioteca universitaria di Varsavia, BUW, offre cataloghi ricchi e aggiornati ${ }^{11}$. Invece

4. Cfr. Floryan, Władysław (a cura di), Drieje literatur europejskich, Warszawa, PWN, 1982, e Gierowski, Józef Andrzej, Historia Włoch, Wrocław, Ossolineum, 1986.

5. Dopo I. Bednarz, che li aveva curati fino al 2000, gli italica polacchi sono a cura di Jolanta Dygul, e dal 2014 la sezione linguistica è affidata a Adrianna Siennicka.

6. BIGLI, http://www.bigli.it/public/pagine/bigli

7. http://pbl.ibl.poznan.pl/dostep/index.php?s=d_biezacy\&f=dzialy\&p_dznadrz=116

8. http://katalog.nukat.edu.pl/search/query?theme=nukat

9. http://alpha.bn.org.pl/

10. http://katalog.pan.pl/webpac-bin/233biblitpl/wgbroker.exe?new+-access+top

11. https:// chamo.buw.uw.edu.pl/search/query?theme=system 
non esiste più lo schedario dei traduttori con il relativo elenco dei libri tradotti, creato al tempo della Polonia Popolare ${ }^{12}$.

Se la frammentarietà dei dati e delle informazioni deve far riflettere alla necessità di costruire banche dati e di ricorrere a metodi quali il distant reading auspicato da Franco Moretti per la world literature, focalizzeremo qui la nostra attenzione su alcuni nuovi fenomeni del mercato editoriale polacco di traduzioni dall'italiano. La cesura che vorremmo proporre risale al fatidico 1989. Prima della caduta del muro di Berlino, il regime aveva monopolizzato in maniera quasi incontestata ${ }^{13}$ il pensiero e la parola, autorizzando e programmando il lavoro degli editori, tutti rigorosamente statali, e determinando la scelta della letteratura da tradurre. Non per altri motivi, se non quelli di ordine ideologico (per esempio, criticare i soprusi del mondo capitalista), nella Polonia Popolare si tradussero subito Una donna di Sibilla Aleramo, i romanzi sulla realtà lavorativa in fabbrica di Matilde Serao, e la letteratura neorealista del dopoguerra che ben si prestava ai fini di propaganda del realismo socialista che accolse molte opere, da L'Agnese va a morire di Renata Viganò e Cristo si è fermato a Eboli di Carlo Levi, a Metello di Pratolini, Uomini e no di Vittorini, fino a Gesù fate luce di Domenico Rea o L'oro di Napoli di Marotta e La ragaz?a di Bube di Cassola (ma non i libri di Beppe Fenoglio).

Per inciso, non è chiaro se la memoria di quei tempi passati, in cui si recepivano con entusiasmo opere come Donnarumma all'assalto di Ottieri e Il padrone di Parise, influenzi oggi la resistenza che si constata in Polonia verso temi frequenti nella produzione italiana degli ultimi anni, ovvero precariato e disoccupazione, i nuovi poveri, dismissione e delocalizzazione di aziende, morti bianche, ecc. Mancano inoltre oggi in Polonia - ad eccezione di due libri di Ornela Vorpsi - traduzioni di scrittori «translingui » in voga in Italia e in altri paesi esteri. Sembra che per essere tradotti in polacco occorra un'identità nazionale forte e che la Polonia non si sia ancora liberata dal complesso storico di un paese che per esistere si deve aggrappare alla propria lingua e letteratura ${ }^{14}$. In tal senso il mercato polacco di oggi registra fenomeni inversi rispetto a quello tede-

12. L'Index translationum dell'Unesco, infine, ha il vantaggio di essere di immediata reperibilità, ma non è aggiornato (si ferma al 2008).

13. Data la censura, esisteva un circuito di libri stampati all'estero e distribuiti clandestinamente.

14. Cfr. "Piccole letterature più o meno globali??", L'Indice dei libri del mese, Torino, 26 gennaio 2013; http://www.lindiceonline.com/index.php/blog/l-indiceallungato/328-piccole-letterature-piu-o-meno-globali?highlight=Y'ToxOntpOjA7czo5OiJzZXJrb3dza2EiO30= 
sco o francese. Solo la non fiction gode di incontestata fortuna editoriale nel paese di Ryszard Kapuściński. Lo confermano le traduzioni dei reportage di viaggio da Chernobyl di Francesco Cataluccio, Lo Stivale meticcio di Stefano Liberti, e Tribù bianche perdute. Viaggio tra i dimenticati di Riccardo Orizio (vincitore del Premio Kapuściński), nonché gli innumerevoli reportage di Tiziano Terzani, per non parlare dell'opera omnia di Fallaci o dei recentissimi Io sono il mercato di Luca Rastello e La leggenda dei monti naviganti di Paolo Rumiz.

Chiusa la parentesi, e tornando al periodo che precede il fatidico 1989, va detto che furono allora accolti bene anche i romanzi di Guido Morselli, presumibilmente per l'anticlericalismo dell'autore, e i racconti di Buzzati per il distacco da politica e ideologia che offriva il realismo magico. Il regime dimostrava inoltre una certa deferenza verso i classici del '900 (forse perché molti autori avevano simpatie a sinistra ${ }^{15}$ ) e ne proponeva in traduzione le principali opere; sono noti da tempo Morante, Sciascia, Moravia, Pavese, Pirandello, Gramsci, Deledda, Calvino, Bassani, Tomasi di Lampedusa, Satta, Dessì, Tobino ${ }^{16}$.

Prima che venisse stroncata la spina dorsale dei polacchi con la legge marziale del 1981 ( $\mathrm{ma}$ in questi anni iniziarono a essere pubblicate le opere di Tabucchi), la Polonia Popolare aveva vissuto, specie nei primi anni Settanta, un periodo di relativo disgelo: appaiono le traduzioni della difficile prosa sperimentale di Gadda e di Palazzeschi, e al contempo nasce, nel 1971, la benemerita rivista Literatura na świecie che diffonde in Polonia le letterature straniere, compresa quella italiana, anche pubblicando brani in polacco ${ }^{17}$.

15. L'orientamento ideologico degli scrittori italiani venne criticato a suo tempo dallo scrittore polacco stabilitosi a Napoli, Gustav Herling-Grudziński, marito di Elena Croce, il quale biasimava perfino uno scrittore come Calvino, e si lamentava di essere emarginato dagli intellettuali e artisti italiani, tutti comunisti, perché non potevano riconoscere quanto aveva scritto in Un mondo a parte (pubblicato in Italia solo nel 1951, e in Polonia soltanto dopo il 1989).

16. Argutamente notava lo scrittore di fantascienza polacco Stanisław Lem che nell'Unione Sovietica tutti leggevano Dostoevskij perché non v'era altro da leggere. Cfr. UgniewsKa, Joanna, Przektady zpowojennej literatury utoskiej po 1989 roku, in SERKOwSKA, Hanna (a cura di), Literatura ntoska w toku, Wrocław, Ossolineum, 2006, pp. 215-219. V. anche UgNiewskA, Joanna, Traduzioni della letteratura contemporanea italiana in Polonia in Lingua e letteratura italiana in Europa, Le Monnier, Firenze, 1990; ŻABOKLICKI, Krzysztof, "La letteratura italiana in Polonia", L'inventario, n. 13, 1985, e ID., La presenza della letteratura italiana in Polonia, oggi, Firenze, Leo Olschki, 1991; infine MiszALSKA, Jadwiga et alii, Od Boccaccia do Eco. Wtoska proza narracyjna w Polsce (od XVI do XXI wieku), Kraków, Collegium Collombinum, 2011.

17. I numeri monografici dedicati alla letteratura italiana sono: 4/1993; 12/1997; 3-4/2005; 3-4/2009; 1-2/2013. Hanno contribuito alla diffusione della letteratura italiana in Polonia anche il bimensile Literatura na świecie, il trimestrale Zeszyyty Literackie, 
Voltiamo pagina. Dal 1989, sparito il circuito clandestino, viene messo in circolazione un numero entropicamente crescente di libri ${ }^{18}$, in un mercato regolato dagli stessi meccanismi di portata globale: sovra-offerta di libri destinati a vita breve, poi all'oblio. Per tutto il lungo periodo di transizione, di cui oggi è erede il sistema polacco, hanno continuato a esistere seppur con alterne vicende prestigiose case editrici, risalenti al dopoguerra della Polonia Popolare, come la Państwowy Instytut Wydawniczy, PIW, la Spółdzielnia Wydawnicza "Czytelnik" e la Wydawnictwo Literackie, specializzate nelle traduzioni della migliore letteratura internazionale. Vi si sono man mano affiancati altri editori, rendendo caleidoscopico il quadro da indagare. Tra le oltre trentamila case editrici presenti oggi in Polonia, quelle che pubblicano i libri tradotti (anche dall'italiano) sono una buona ventina ${ }^{19}$. Le ragioni di tale frantumazione, s'è detto, spesso non trascendono i condizionamenti economici, al punto che si ha l'impressione che il mercato dei libri in traduzione viva una vita autonoma rispetto alle richieste e alle aspettative dei lettori, mentre spesso finisce per influenzarli e appiattirne i gusti.

A uno sguardo di insieme sui titoli italiani in traduzione, si ha l'impressione di una mancanza di criteri univoci, e perfino di un caotico tentativo di fare di tutto, perché molto è ancora da fare. Il proliferare delle case editrici porta i suoi frutti. E gli interessi economici portano gli editori a guardare anche alla letteratura più contemporanea. Bisogna dire che, grazie ai contatti diretti con l'editoria francese, se uno scrittore è stato tradotto in Francia (la regola vale per Erri De Luca, Milena Agus e Michela Murgia), la traduzione in polacco è garantita.

Un elemento di continuità rispetto al periodo comunista è la figura autorevole del traduttore, specie se proveniente dall'ambiente accademico. Ai tempi della Polonia Popolare due bravissime traduttrici, Zofia Ennstowa e Barbara Sieroszewska, erano in grado di valutare e consigliare gli editori. Dal 1989 il

altre riviste letterarie come Twórczość e Życie Literackie, e riviste popolari come Przekrój o Prayjaciótka.

18. Dal 1989, per circa un decennio, si è assistito a una fase protratta di transizione. Il capitalismo frenetico, stabilitosi poi di colpo, benché abbia contribuito a migliorare il tenore di vita, ha avuto un sicuro contraccolpo sulla cultura, limitando la traduzione delle letterature straniere, anche perché, abolita la censura, i lettori erano curiosi di leggere le voci indigene, che finalmente potevano esprimersi. D'altra parte, la nuova industria editoriale proponeva autori e titolo consacrati dal successo di massa all'estero.

19. Cfr. in particolare: Muza, Noir sur Blanc, Prószyński i S-ka, Prószyński Media, Świat KsiąŻki, Zysk i S-ka, W.A.B., Czarne, Sonia Draga, Rebis, Wydawnictwo Dolnośląskie, Adam Marszałek, Znak, Atut, Albatros Andrzej Kuryłowicz, Amber, Vesper, Między słowami, Smak Słowa, Twój Styl, Znak. Literanova, Marginesy, Czwarta strona, KsiąŻkowe Klimaty, Czarna Owca. 
novero di traduttori dall'italiano si è andato convulsamente ampliando, e a nomi da tempo presenti sul mercato - Bernhard-Kralowa, Kasprzysiak, Żaboklicki, Ugniewska, Salwa, Wasilewska, Kabac, Kasprzysiak - si sono aggiunti quelli di Osmólska-Mętrak, Pawłowska-Zampino, Woźniak, Łukaszewicz. Ai traduttori della generazione dei maestri dobbiamo le traduzioni non solo dei grandi romanzieri del passato e del presente (Pasolini, Bassani, Tomasi di Lampedusa, Tabucchi, Magris), ma anche di molta importante saggistica di Calasso, Ceronetti, Camporesi, Savinio, Eco, Agamben.

Una specificità del mercato polacco dopo il 1989 è la predilezione degli editori per alcuni autori e traduttori. Per esempio tutte le opere di Tabucchi sono uscite presso la Czytelnik, tradotte da Ugniewska; Camilleri e la Jaeggy sembrano monopolizzati da Noir sur Blanc; casa editrice W.A.B. pubblica Mazzucco, Carofiglio e Agus; Świat Książki, la Murgia, mentre Muza pubblica Baricco, Ammaniti e Moccia. Di recente Sonia Draga si è specializzata in Ferrante, Saviano e Mazzantini, ma pubblica anche Catozzella, Costantini, Simoni. Col tempo il quadro si è venuto complicando e altre case editrici hanno proposto altri autori: Wydawnictwo Dolnośląskie ha puntato su libri collaudati e di sicuro effetto come La pelle di Malaparte e Lettera a un bambino mai nato di Fallaci, ma anche su Ė una vita che ti aspetto di Volo; Albatros ha scelto il clamoroso Cento colpi di spazzola di Melissa P.; Dodo Editor ha proposto La briganta di Cutrufelli; Petra ha pubblicato I dodici abati di Challant di Mancinelli, e Draft Publishing Caos calmo di Veronesi. La prestigiosa e ambita Czarne (nata nel 1996 e legata allo scrittore polacco contemporaneo più tradotto all'estero, Andrzej Stasiuk), specializzata in non fiction, reportage di viaggio e saggistica, pubblica Agnello Hornby, Cataluccio, Vorpsi, Liberti, Orizio, Rastello e Rumiz. Solo Terzani non appartiene in via esclusiva a un unico editore e traduttore.

Per quanto riguarda le scrittrici, occorre dire che anche prima del 1989 si sono tradotte in polacco le opere di Fallaci, ma così non è stato per le più grandi scrittrici del Novecento. Di Natalia Ginzburg sono stati tradotti Caro Michele (1976) e Tutti i nostri ieri (2014), di Elsa Morante solo due romanzi, L'isola di Arturo (1960) e Menrogna e sortilegio (1968). Dopo il 1989 nulla più è uscito di Alba De Céspedes, che in compenso ebbe un'enorme fortuna negli anni precedenti. Poco prima della Ferrante Fever e dei romanzi-sceneggiatura di Margaret Mazzantini, sono state tradotte più opere di Fleur Jaeggy e Agnello Hornby, nonché di Tamaro, Mazzucco, Maraini e Morandini, cui vanno aggiunti innumerevoli libri di Sveva Casati Modignani, e un'opera di Mancinelli (I dodici abati di Challant), una di Pariani (Stradurione), una di Cutrufelli (La briganta) e una di Petrignani (La scrittrice abita qui). 
Affinché chi legge possa farsi un'idea della letteratura iper-contemporanea, notiamo che a queste liste mancano molti nomi, tra cui Genna, Pascale, Parrella, Scurati, Scarpa, Affinati. Lagioia, Trevi, Vasta, Avallone, Wu Ming, e perché no Mariolina Venezia, Laura Pugno e Rosa Matteucci. Ancora più acutamente si avverte l'assenza di scrittori di generazioni precedenti, come Parise, Volponi, Balestrini, La Capria, Tondelli o Celati ${ }^{20}$, mentre due grandissimi, Landolfi (Il mare delle blatte) e Manganelli (Centuria) sono stati per la prima volta tradotti solo nel 2016.

Vanno invece considerati a parte, e nell'ottica del best seller, le cui leggi ha di recente spiegato Stefano Calabrese ${ }^{21}$, alcuni dei nuovi autori di world fiction, come Ferrante, Saviano, Camilleri, Moccia, e più di recente Mazzantini. Quest'ultima, tradottissima in Polonia, è vincitrice di importanti premi letterari, ma soprattutto ha al suo attivo diverse sceneggiature e romanzi che si leggono come tali e vengono adattati al cinema. Il film ha un grande impatto per la promozione del libro, in Polonia come altrove; un romanzo trasposto in film limita i rischi, l'acquisto dei diritti per la traduzione è sicuro. Un altro requisito che Calabrese individua a carico dei best seller è la serialità richiesta per presidiare il mercato librario (vedi la tetralogia di Elena Ferrante) e fidelizzare il lettore di cui si cerca di soddisfare il bisogno di immersione. Il benessere che genera la lettura causa dipendenza e binge reading. La posta in gioco nei tempi astorici che viviamo non è la sopravvivenza del testo: il libro ha le stesse date di scadenza dei prodotti di mercato, i suoi tempi di permanenza sono ridotti al massimo a tre mesi.

Un'altra riprova del fatto che i meccanismi che presiedono al mercato editoriale polacco di oggi non sono diversi da quelli globali è la figura del critico letterario, che da importante attore e mediatore del mondo editoriale, quando non del tutto eclissata, è passata in secondo piano. Un tempo arbitro e intenditore del bello stile, è stato soppiantato da lettori non professionisti che su vari blog ne hanno usurpato le funzioni, e da scrittori che si recensiscono a vicenda. Il mercato ha imparato benissimo a fare a meno di critici letterari e recensori. Nel suo articolo "Come nascono i bestseller", Milena Rachid Chehab individua

20. Dieci anni fa, pensando al modo alquanto caotico in cui al pubblico polacco venivano presentate le opere letterarie italiane, abbiamo ideato una collana di cui sono usciti due volumi dal titolo Literatura wtoska w toku (Scritture in corso 1/2006 e 2/2010), con saggi critici su scrittori allora in Polonia pressoché ignoti, come Ammaniti, Baricco, Benni, Celati, Consolo, Vassalli, Andrea Baiani, Camporesi, Citati, Covacich, De Carlo, De Luca, Maurensig, Nove, Scurati, Veronesi, Bruck, Jarre, Jaeggy, Merini, Morazzoni, Capriolo, Ferrante. L'iniziativa andrebbe rinnovata.

21. Calabrese, Stefano, Anatomia del best seller. Come sono fatti i romanzi di successo, Roma-Bari, Laterza 2015, pp. 85-99 e 131-160. 
tra i fattori di successo il doppio ruolo dei $\operatorname{blog}^{22}$. Da una parte, l'autore è incoraggiato a gestire un blog, una pagina Facebook o altri social per essere in contatto costante con i potenziali acquirenti dei suoi libri. Dall'altra, l'editore decide se acquistare o meno il manoscritto in base al blog (per esempio, lubimyczytac. pl, modesto parente del Goodreads.com) o affidandosi al parere dello scout (spesso un ex editore o libraio) che legge e consiglia i manoscritti.

Tra le manifestazioni che aiutano a promuovere le traduzioni della letteratura italiana in Polonia un ruolo importante spetta alle fiere del libro, organizzate più volte nel corso dell'anno nelle maggiori città, ai vari festival e premi letterari che prevedono anche la categoria "traduzione/traduttore". Un fatto eloquente a conferma della presenza consolidata e in costante crescita del libro italiano in Polonia è l'istituzione nel 2014 del premio letterario "Leopold Staff” dedicato esclusivamente a cultura e letteratura italiana. Nel 2016 la giuria ha premiato, oltre ad autori, cineasti, scrittori, i seguenti traduttori: Zofia Ernst (traduttrice in memoriam), Elżbieta Jogałła (Magris), Natalia Mętrak-Rudy (Fo) e Katarzyna Skórska (Mazzucco) e Janna Wajs (Vanni Bianconi).

Il dopo 1989 ha portato grandi cambiamenti anche per la poesia. Il mercato libero, l'apertura verso l'estero e il libero accesso a ogni tipo d'informazione hanno progressivamente marginalizzato la cultura alta. Il mercato letterario spezzettato in molti centri ha modificato sia la situazione dei poeti (privandoli di importanza) che le aspettative dei lettori (la decentralizzazione geografica delle istituzioni culturali ha favorito l'esordio dei poeti e il loro contatto col pubblico). Queste tendenze sono ben visibili nel mercato traduttivo che dopo il 1989 si è concentrato soprattutto sulla prosa. La posizione dell'italiano rimane costante: seppur popolarissimo, non ha mai fatto parte delle lingue più tradotte e oggi rimane indietro rispetto a lingue di maggiore impatto, inglese, tedesco, francese, spagnolo. Eppure, dopo il 1989 il numero delle traduzioni di poesia italiana è notevolmente aumentato senza però influire sulla loro ricezione: sono apparsi, infatti, piccoli editori che, incoraggiati dai traduttori, hanno pubblicato poeti italiani raggiungendo solo una piccola fetta di pubblico. Inoltre le case editrici più grandi, come PIW e Wydawnictwo Literackie, hanno perso l'interesse per la poesia italiana dimostrato sino alla fine degli anni Ottanta, limitando la propria attività alla ristampa delle opere classiche tradotte dai migliori traduttori del '900: Dante tradotto da Edward Porębowicz, Petrarca da Jalu Kurek, Leonardo da Vinci da Leopold Staff. Dopo il 1989, la poesia italiana è stata

22. Rachid Chehab, Milena, "Skąd się biorą bestsellery", Ksią̇̇ki. Magazynn do caytania, n. 2, 2016, pp. 15-18. 
accolta in piccole case editrici come Maj, Teta Veleta, Świat Literacki, Spółka Wydawnicza Heliodor, Austeria, Anagram, Biuro Literackie, e grazie a traduttori che, dopo Eugeniusz Kabatc e Halina Kralowa, si sono fatti i grandi divulgatori della cultura italiana in Polonia, come Jarosław Mikołajewski, Paweł Krupka, Cezary Geroń, Monika Woźniak, Monika Gurgul, Jadwiga Miszalska ${ }^{23}$, Monika Surma-Gawłowska, Aleksander Sambor.

Nonostante si traduca sempre di più (seppur con tirature più basse), nella pubblicazione della poesia italiana si rinvengono enormi lacune, in particolare per quanto riguarda la poesia dei secoli che precedono il Novecento. Oltre alle ristampe delle vecchie traduzioni di Petrarca, appaiono le nuove traduzioni di Agnieszka Kuciak (1992) e di Andrzej Lewandowski (2004). Nel 2005 esce l'intero Canzoniere. Il poeta italiano più noto in Polonia resta comunque Dante Alighieri. Dopo il 1989 escono su rivista traduzioni parziali di Stanisław Barańczak, Jarosław Mikołajewski e Maciej Froński24. Nel 1992 Tomasz Łubieński pubblica 15 canti della Divina Commedia, nel 1993 l'Inferno è tradotto da Bernard Antochewicz. Nel 2000 esce Vita Nuova tradotta da Edward Porębowicz e un anno dopo tutta la Divina Commedia in traduzione di Alina Świderska. Negli anni 2002-2004 per i tipi della Biblioteka Telgte viene pubblicata la controversa - perché a tratti colloquiale e scurrile - traduzione di Agnieszka Kuciak. Invece fino al 2005, quando esce Przed Petrarkq. Antologia traynastowiecznej poerji utoskiej a cura di Monika Woźniak e vari traduttori, era mancata in Polonia un'antologia di poesia italiana del Duecento. Un anno dopo appare un'antologia della poesia italiana dal '500 all"800 (Antologia polskich pręektadów poezji utoskiej. Od wieku XVI do końca XIX stulecia) a cura di Monika Gurgul e Jadwiga Miszalska. Molto amati sono stati Michelangelo Buonarotti, tradotto da Jerzy Kolankowski (1998) e Leapold Staff (2003), e Giambattista Marino le cui Rime sono tradotte da Piotr Kostka (1996); più di recente La strage degli innocenti viene pubblicata a cura di Radosław Rusnak (2012). Nel 2009 la casa editrice Maj pubblica i Canti di Giacomo Leopardi a cura di Joanna Ugniewska (con traduzioni di Jadwiga Dickstein-WieleŻyńska, Stanisław Kasprzysiak e Jarosław Mikołajewski). Un anno dopo esce una selezione dell'Infinito tradotto da Grzegorz Franczak. Numerose traduzioni vengono pubblicate anche in rivista: così Il Cantico di Frate Sole di San Francesco, traduzione di

23. Si veda Gurgul, Monika, Miszalska, Jadwiga, Antologia polskich præeektadów poezji wtoskiej, Kraków, Collegium Columbinum, 2005.

24. V. Zessyty Literackie, n. 33, 2015, pp. 47-50; Arkusz, n. 4, 2001, p. 8; Magasyn Literacki, n. 12, 1999, p. 60. 
Roman Brandstetter, apparsa sulla stampa cattolica, il che limita la notorietà del poeta $^{25}$. Le traduzioni di altri poeti dei secoli passati appaiono saltuariamente in rivista e hanno poco seguito.

Molto più presente oggi in Polonia è la poesia contemporanea, grazie soprattutto a Jarosław Mikołajewski che in pochi anni ha tradotto numerose opere, e ha pubblicato nel 1997 un'antologia della poesia italiana novecentesca, Radość rozbitków (Allegria di naufraghi), con testi di Saba, Campana, Montale, Quasimodo e altri. Nel 1989 escono le poesie di Sandro Penna, con vari traduttori (Driwna radość Życia, Una strana gioia di vivere). Nel 1994 Świat Literacki pubblica Mario Luzi, tradotto da Jarosław Mikołajewski e Cezary Gieroń (Szcześlinwi, k.tórayy jestéście w rucbu); per i tipi di Teta Veleta, nel 1999 esce Bestemmia di Pier Paolo Pasolini, tradotta da Mikołajewski, e nel 2001 la raccolta di poesie Saba. Triest i poeta, di vari traduttori.

Più di recente, si assiste al ritorno in auge di grandi poeti italiani (nel $2013 \mathrm{e}$ 2014, l'Istituto Italiano di Cultura di Cracovia, l'editore Austeria Klezmerhojs e Jarosław Mikołajewski collaborano all'edizione polacca delle poesie di Pavese, Ungaretti e Primo Levi), ma anche alla pubblicazione di poeti italiani meno noti. Per esempio, nel 2006, escono Il tempo successivo di Carlo Cipparrone e Via delle nuvole di Franco Dionesalvi, tradotti da Paweł Krupka, nonché l'antologia della poesia siciliana di Saragei Antonini e altri, Mój driadek to atlas, ojciec čztowiek$r y b a^{26}$, tradotta da Paweł Krupka che due anni dopo tradurrà le poesie di Filippo Senatore Noi e i ragazzi del Portnoy di Milano. Nello stesso anno appare Il Miele di Tonino Guerra tradotto da Jolanta Dygul, e nel 2009 la raccolta Andate e ritorni di Ugo Piscopo, di nuovo curata da Paweł Krupka.

Per concludere, se certamente il libro italiano registra attualmente un forte successo in Polonia - com'è dimostrato dal numero crescente di autori tradotti e di traduttori, e dalla creazione di festival e premi letterari - tanto per la prosa quanto per la poesia prevale una sensazione di spaesamento di fronte alle condizioni caotiche del mercato editoriale polacco: manca una politica editoriale che faccia emergere una tendenza, prova ne sia il modo in cui si stampano in rivista $i$ versi dei più grandi poeti novecenteschi (Saba, Montale, Ungaretti, Caproni, Campana, Merini) affianco a poeti più giovani o poco noti come

25. Miszalska, Jadwiga et al., Od Dantego do Fo. Włoska poezja i dramat w Polsce (od XVI do XXI wieku), Kraków, Collegium Columbinum, 2007, p. 31.

26. Antonini, Saragei et al., Mój driadek to atlas, ojciec cztowiek - ryba: antologia poezji włoskiej., trad. P. Krupka et al., Rzeszów, Podkarpacki Instytut KsiąŻki i Marketingu, 2006. 
Nicola Gardini, Matteo Zattoni e Francesca Moccia ${ }^{27}$. Le poesie di questi ultimi, presentate singolarmente a un lettore che non conosce il contesto, rimangono come sospese nel vuoto.

Hanna SERKOwSKA Università di Varsavia

Katarzyna Misiewicz Università di Varsavia

27. Cfr. Literatura na Ś wiecie, n. 4, 1993; Kresy, n. 40, 1999 e n. 44, 2000; Nowa Okolica Poetón, n. 6, 2000, e Arkuš, n. 12, 2000. 
